\title{
Tratamiento endoscópico de la obstrucción biliar en cáncer de páncreas no resecable
}

\author{
Alejandra Tepox-Padrón y Félix I. Téllez-Ávila* \\ Departamento de Endoscopia Gastrointestinal, Instituto Nacional de Ciencias Médicas y Nutrición Dr. Salvador Zubirán, Ciudad de México, México
}

\section{Resumen}

El cáncer de páncreas es un problema mundial de salud. La mayoría de los pacientes al diagnóstico no son candidatos a tratamiento curativo, por lo que finalmente desarrollarán complicaciones como la obstrucción biliar y requerirán tratamiento paliativo. Actualmente la endoscopia es la piedra angular del tratamiento de la obstrucción biliar maligna, debido a que se asocia a menor morbilidad comparada con el drenaje percutáneo y la cirugía. El drenaje biliar por colangiopancreatografía retrógrada endoscópica (CPRE) mediante prótesis metálicas autoexpandibles debe considerarse la primera opción de tratamiento. En casos en los que la CPRE falla, se deberá preferir el drenaje biliar guiado por ultrasonido endoscópico (DB-USE) como tratamiento de rescate. A pesar de que existe cada vez más evidencia de que el DB-USE puede sustituir a la CPRE como tratamiento inicial en estos pacientes, aún se necesitan más estudios.

Palabras clave: Colangiopancreatografía retrógrada endoscópica. Ultrasonido endoscópico. Cáncer de páncreas. Obstrucción biliar maligna.

\section{Endoscopic treatment of biliary obstruction in unresectable pancreatic cancer}

\begin{abstract}
Pancreatic cancer is a worldwide health problem. At diagnosis, most of them are not suitable for curative treatment, so they will eventually develop complications such as biliary obstruction, therefore they will require palliative treatment. Endoscopy currently forms the cornerstone in the treatment of malignant biliary obstruction, due to its lower morbidity compared with both percutaneous drainage and surgery. Endoscopic retrograde cholangiopancreatography (ERCP) biliary drainage by using self-expandable metal stent should be considered the first-line treatment. In patients with failed ERCP, endoscopic ultrasound-guided biliary drainage (EUS-BD) ought to be the preferred rescue therapy. Although there is increasing evidence that EUS-BD can replace ERCP for the primary treatment of unresectable pancreatic cancer, further studies are needed.
\end{abstract}

Key words: Endoscopic retrograde cholangiopancreatography. Endoscopic ultrasound. Pancreatic cancer. Malignant biliary obstruction.

Correspondencia:

*Félix Téllez-Ávila

E-mail: felix.telleza@incmnsz.mx
Fecha de recepción: 11-06-2020

Fecha de aceptación: 15-06-2020

DOI: 10.24875/END.M20000179
Disponible en internet: 00-00-2020

Endoscopia. 2020;32(2):55-61

www.endoscopia-ameg.com

0188-9893/() 2020. Asociación Mexicana de Endoscopia Gastrointestinal y Colegio de Profesionistas, A.C. Publicado por Permanyer. Éste es un artículo open access bajo la licencia CC BY-NC-ND (http://creativecommons.org/licenses/by-nc-nd/4.0/). 
Actualmente el cáncer de páncreas ocupa el decimosegundo lugar en frecuencia en el mundo y el séptimo lugar en mortalidad de acuerdo con el Global Cancer Observatory (GLOBOCAN 2018, https://gco.iarc. $\mathrm{fr} /$ ). Desafortunadamente, la mayoría de los pacientes al diagnóstico se presentan con enfermedad localmente avanzada o metastásica (80-85\%) y solo entre un 15$20 \%$ son candidatos a tratamiento quirúrgico ${ }^{1,2}$.

La obstrucción sintomática de la vía biliar se presenta en el $80 \%$ de los pacientes con cáncer de páncreas y su presentación impacta directamente la calidad de vida de los pacientes ${ }^{3,4}$, aunado al riesgo latente de desarrollar colangitis, malabsorción, prurito y falla hepática ${ }^{5}$.

Actualmente, el tratamiento endoscópico es la piedra angular en la paliación de la obstrucción biliar en cáncer de páncreas, como se describirá en esta revisión.

\section{Comparación entre drenaje quirúrgico, drenaje percutáneo y drenaje endoscópico}

Durante muchos años, el tratamiento paliativo de la obstrucción biliar fue la cirugía, sin embargo, actualmente el tratamiento endoscópico es el método de elección para el manejo de estos pacientes ${ }^{3}$. Al comparar directamente el tratamiento quirúrgico frente al tratamiento endoscópico en pacientes con obstrucción biliar maligna, no se han observado diferencias en el éxito técnico y clínico, ni en las complicaciones globales y la mortalidad. Sin embargo, el tratamiento endoscópico se asocia a menor estancia hospitalaria y menor presentación de complicaciones graves. Por otro lado, la cirugía se asocia a menor recurrencia de la obstrucción ${ }^{4,6}$. De acuerdo con estos datos, la cirugía pudiera ser una opción viable en pacientes con buen estado funcional y bajo riesgo quirúrgico.

En el caso del drenaje biliar transhepático percutáneo (DBTP), representa tanto una alternativa como una medida de rescate en el tratamiento de la obstrucción biliar maligna. Al comparar el DBTP frente a la colangiopancreatografía retrógrada endoscópica (CPRE), no se ha observado diferencia en el éxito terapéutico, la mortalidad a 30 días y las complicaciones generales. EI DBTP se ha asociado a menor riesgo de colangitis ${ }^{7,8}$ y menor riesgo de pancreatitis ${ }^{8}$, pero tiene mayor riesgo de migración del catéter de drenaje y hemorragia asociada al procedimiento, sin mencionar el impacto negativo en la calidad de vida al tener un catéter de drenaje externo a permanencia ${ }^{7}$.

En la tabla 1 se muestran los diversos tratamientos para el drenaje biliar en la obstrucción biliar.

\section{Drenaje biliar por colangiopancreatografía retrógrada endoscópica}

\section{Comparación entre prótesis plásticas y metálicas}

El drenaje biliar con intención paliativa mediante el uso de prótesis se asocia a un impacto favorable en la calidad de vida de los pacientes ${ }^{9}$. Existen dos tipos principales de prótesis, que son las plásticas y las metálicas autoexpandibles.

Las prótesis plásticas son económicas y accesibles. La principal desventaja es su tendencia a obstruirse, tanto por su diámetro interno reducido como por la predisposición a formar biofilms bacterianos y lodo biliar, lo que lleva a la recurrencia de la ictericia y el prurito y la posibilidad de desarrollar colangitis 5 . Por otro lado, las prótesis metálicas son más costosas, pero su diámetro interno es mayor, lo que prolonga su tiempo de permeabilidad ${ }^{10}$.

Al comparar las prótesis plásticas con las metálicas autoexpandibles como tratamiento paliativo de la obstrucción biliar maligna, no se ha demostrado diferencia en éxito técnico, clínico y supervivencia del pacientes $^{4,11}$; sin embargo, las prótesis metálicas se asocian a menor desarrollo de complicaciones generales y particulares, como la colangitis y la obstrucción de la prótesis por lodo. Además se asocian a menor recurrencia de la obstrucción biliar y menor necesidad de reintervención, así como a mayor supervivencia libre de síntomas a seis meses ${ }^{11}$.

\section{Costo-efectividad}

En los análisis de costo-efectividad, el tratamiento inicial con prótesis plásticas ha resultado menos efectivo y más costoso en este grupo de pacientes. Sin embargo, la ventaja con el uso de prótesis metálicas disminuye en pacientes con supervivencia menor a seis meses. Finalmente, en pacientes con supervivencia esperada menor a tres meses, la colocación de una prótesis plástica es la estrategia más costo-efectiva ${ }^{5,12}$.

Recientemente, se describió el uso de una nueva prótesis plástica de $12 \mathrm{Fr}$. Los resultados de su uso mostraron una tendencia a favor de la duración de la permeabilidad de la nueva prótesis y menor riesgo de recurrencia de la obstrucción comparada con las prótesis de $10 \mathrm{Fr}$. El riesgo de recurrencia de la obstrucción no fue diferente al compararla con las metálicas, por lo que su uso pudiera ser una alternativa efectiva y de menor costo comparada con el uso de prótesis metálicas ${ }^{13}$. 
Tabla 1. Tratamiento primario de la obstrucción biliar por cáncer de páncreas no resecable. Comparación entre drenaje quirúrgico, percutáneo y endoscópico

\begin{tabular}{|c|c|c|c|c|}
\hline Técnica & Éxito terapéutico (\%) & Complicaciones (\%) & $\begin{array}{l}\text { Necesidad de } \\
\text { reintervención (\%) }\end{array}$ & $\begin{array}{l}\text { Estancia hospitalaria } \\
\text { (días/ rango) }\end{array}$ \\
\hline $\begin{array}{l}\text { Drenaje quirúrgico } \\
\text { Glazer, et al., 2014 }\end{array}$ & 89.5 & 29 & 3.1 & $21.8 \pm 5$ \\
\hline $\begin{array}{l}\text { DBTP } \\
\text { Zhao, et al., } 2015^{8} \\
\text { Pinol, el at., 2002 } \\
\text { Tellez, et al., } 2018^{15}\end{array}$ & $48.4-93.9$ & $12.9-67$ & 43 & $11.9 \pm 8.5$ \\
\hline $\begin{array}{l}\text { CPRE (PM) } \\
\text { Paik, et al., 2018 } \\
\text { Bang, et al., 2018 } \\
\text { Tellez, et al., 2020 }\end{array}$ & 91.2 & 14.7 & 42.6 & $5 \pm 1$ \\
\hline $\begin{array}{l}\text { USE } \\
\text { Paik, et al., } 2018^{16} \\
\text { Bang, et al., 2018 } \\
\text { Tellez, et al., 2020 } \\
\text { Hathorn, et al., 2019 }\end{array}$ & 97 & 21.2 & 7 & $4(3-5)$ \\
\hline
\end{tabular}

DBTP: drenaje biliar transhepático percutáneo; CPRE: colangiopancreatografía retrógrada endoscópica; PM: prótesis metálicas; USE: ultrasonido endoscópico.

\section{Comparación entre prótesis metálicas no cubiertas y cubiertas}

Actualmente se dispone de prótesis metálicas no cubiertas, cuyo riesgo principal es la oclusión por crecimiento dentro de la prótesis, el cual ocurre en un 20 a $50 \%$ de los pacientes después de ocho meses de su colocación ${ }^{20}$. También se dispone de prótesis cubiertas, tanto totales como parcialmente cubiertas; estas se han asociado a mayor riesgo de migración, desarrollo de colecistitis y pancreatitis, y mayor disfunción debida a la formación de lodo, ya que la cobertura provee una superficie de adherencia para el desarrollo de biofilms bacterianos ${ }^{10,21}$.

De acuerdo con el metaanálisis realizada por Tringali, et al..$^{22}$ (11 estudios; 1,272 pacientes), en el que se comparó el uso de prótesis cubiertas frente a no cubiertas para el tratamiento de las estenosis malignas distales de la vía biliar. No se encontraron diferencias en la falla de la prótesis, ni en mortalidad. Sin embargo, se observó beneficio con el uso de las prótesis cubiertas en la supervivencia de la prótesis y la supervivencia de los pacientes. Pero su uso se asoció a mayor riesgo de migración, crecimiento del tumor en los extremos de la prótesis y formación de lodo. El riesgo de colecistitis, colangitis, pancreatitis, perforación o sangrado entre ambos grupos fue similar. Con base en esto, no se descarta la posibilidad de que el uso de prótesis metálicas cubiertas sea superior a las no cubiertas en términos de supervivencia de la prótesis y de los pacientes, sin embargo su uso se ve limitado por su mayor riesgo de migración y formación de lodo.

Cabe mencionar el estudio multicéntrico aleatorizado de Kitano, et al. ${ }^{23}$, en el que se evaluó el uso de las prótesis parcialmente cubiertas comparado con no cubiertas. Se observó menor riesgo de disfunción y, por consiguiente, mayor duración en la permeabilidad de las prótesis parcialmente cubiertas. La principal causa de disfunción fue debida al crecimiento de tumor dentro de las prótesis no cubiertas (25\%), mientras que la formación de lodo fue la más frecuente en el caso de las parcialmente cubiertas (18.3\%); no se observó migración en ninguno de los dos grupos ${ }^{23}$.

En cuanto al riesgo de colecistitis, el principal factor asociado a su desarrollo es la invasión simultánea del tumor al conducto cístico y su obstrucción al colocar la prótesis cubierta, por lo que en caso de determinarse este factor se deben preferir las prótesis no cubiertas. El riesgo de pancreatitis aguda parece estar asociado a la mayor fuerza axial de la prótesis y a la obstrucción del conducto pancreático principal al colocar la prótesis ${ }^{24}$. La presencia de estas complicaciones no ha sido significativamente mayor en el grupo de las prótesis cubiertas ${ }^{10,22}$.

Debido a que el tratamiento actual del cáncer de páncreas ha incrementado la supervivencia de los pacientes con enfermedad no resecable y metastásica, se han intentado múltiples estrategias para mejorar el tiempo 
de permeabilidad de las prótesis y disminuir su migración. Así, se han evaluado prótesis con propiedades antimigración ${ }^{25}$, prótesis con sistema antirreflujo ${ }^{26}$, prótesis de mayor diámetro $(12 \mathrm{~mm})^{27}$ y prótesis con membranas liberadoras de fármacos quimioterápicos ${ }^{28}$, todas ellas con resultados desalentadores. La colocación transpapilar de las prótesis parece ser efectiva en disminuir la migración y la recurrencia de la obstrucción, pero su uso se reserva para pacientes con cáncer de páncreas cuya estenosis se encuentra a más de $20 \mathrm{~mm}$ por arriba de la papila, por el riesgo de crecimiento del tumor en el extremo distal ${ }^{29}$.

El uso secuencial de ablación por radiofrecuencia endobiliar endoscópica y colocación de prótesis metálicas ha demostrado mejorar el tiempo de permeabilidad de las prótesis, al igual que la supervivencia de los pacientes ${ }^{30}$. Aunque su estudio en el cáncer de páncreas es escaso ${ }^{31}$.

\section{Utilidad del ultrasonido endoscópico en obstrucción biliar maligna}

La CPRE actualmente se considera el método de tratamiento estándar para el manejo de la obstrucción biliar y está asociada a tasas de éxito mayores del $90 \%{ }^{32}$. Sin embargo, en pacientes con cáncer de páncreas avanzado, la canulación del conducto puede resultar imposible por invasión tumoral, al igual que avanzar el duodenoscopio hacia la segunda porción de duodeno; además, estos pacientes pueden presentar anatomía modificada quirúrgicamente.

En estos casos, el drenaje biliar se puede lograr por vía percutánea, aunque actualmente el drenaje biliar guiado por ultrasonido endoscópico (DB-USE) ha ganado terreno, porque es un procedimiento menos invasivo y a fisiológicamente no altera la capacidad de absorción de nutrientes ni genera pérdida de electrólitos ${ }^{33}$.

Comparado con el DBTP, el DB-USE ha demostrado tener éxito técnico similar ${ }^{34,35}$, mayor éxito clínico ${ }^{15,36,37}$, menor incidencia de eventos adversos, particularmente moderados y graves, menor necesidad de reintervención y menores costos asociados ${ }^{14,15,36,38}$. Otras ventajas que ofrece el DB-USE es que puede realizarse en la misma sala de procedimientos, si es que falla la CPRE, y evita los inconvenientes técnicos y cosméticos del drenaje externo.

Entre las técnicas para realizar el DB-USE se encuentran el rendezvous, la colocación anterógrada de prótesis y los transluminales (transgástricas 0 transduodenales) $)^{15,35,39}$.
En la técnica de rendezvous se requiere que la papila sea accesible por vía endoscópica. Se punciona la vía biliar y se avanza una guía a través de la papila hasta el duodeno, posteriormente se retira el ultrasonido endoscópico y se introduce el duodenoscopio para continuar la CPRE de manera habitual ${ }^{39}$. Esta técnica puede intentarse como rescate previo a las técnicas transluminales $34,38,40$.

En la técnica de colocación anterógrada de la prótesis, se avanza una guía hasta el duodeno a través de la vía biliar intrahepática o el conducto biliar común y posteriormente se avanza la prótesis metálica hasta franquear la papila. El inconveniente de estas técnicas es que no se elimina el riesgo de pancreatitis ${ }^{39,41}$.

La técnica transmural puede realizarse por vía transgástrica 0 transduodenal. Las más comúnmente usadas son la hepaticogastrostomía (HGS) y la coledocoduodenostomía (CDS). La HGS es útil en aquellos pacientes en los que no se puede acceder al conducto biliar común, por lo que se accede desde las ramas intrahepáticas izquierdas. Mientras que en la CDS se accede al conducto biliar común generalmente a nivel del bulbo duodenal ${ }^{39}$.

EI DB-USE, de manera global, alcanza porcentajes de éxito técnico del $91.5 \%$, éxito clínico del $87 \%$ y necesidad de reintervención del $6.5 \%$. Sin embargo, está asociado a un porcentaje importante de eventos adversos $(17.9 \%)$, siendo los más frecuentes la fuga biliar $(4.1 \%)$, la migración de la prótesis $(3.9 \%)$, la infección $(3.8 \%)$, el sangrado $(3.5 \%)$ y el pneumoperitoneo $(3.3 \%)^{42}$. Además, a seis meses el $95 \%$ de las prótesis permanecen permeables y a 12 meses el $85 \%$, siendo el abordaje transpapilar el que menos disfunción presenta comparado con el rendezvous y el abordaje anterógrado ${ }^{43}$.

La HGS y la CDS parecen ser equivalentes en su éxito técnico, éxito clínico y duración del procedimiento ${ }^{40,44}$. La presentación de eventos adversos parece ser similar en ambas modalidades ${ }^{40,44} \mathrm{O}$ incluso menor en el grupo de CDS ${ }^{45}$.

El tipo de prótesis utilizada en los drenajes transmurales está directamente relacionado con el desenlace. El uso de prótesis metálicas autoexpandibles alcanza un éxito técnico del $94.1 \%$, éxito clínico del $96.7 \%$ y eventos adversos en el $8.2 \%$. La principal desventaja del uso de prótesis plásticas es la mayor incidencia de fuga biliar ${ }^{46,47}$. En el caso particular de la CDS realizada con prótesis de aposición luminal con electrocauterio, permite simplificar los pasos. Su uso logra el éxito técnico en el $95.7 \%$, éxito clínico en el $95.9 \%$ y parece reducir los eventos adversos al $5.2 \%{ }^{48}$. 


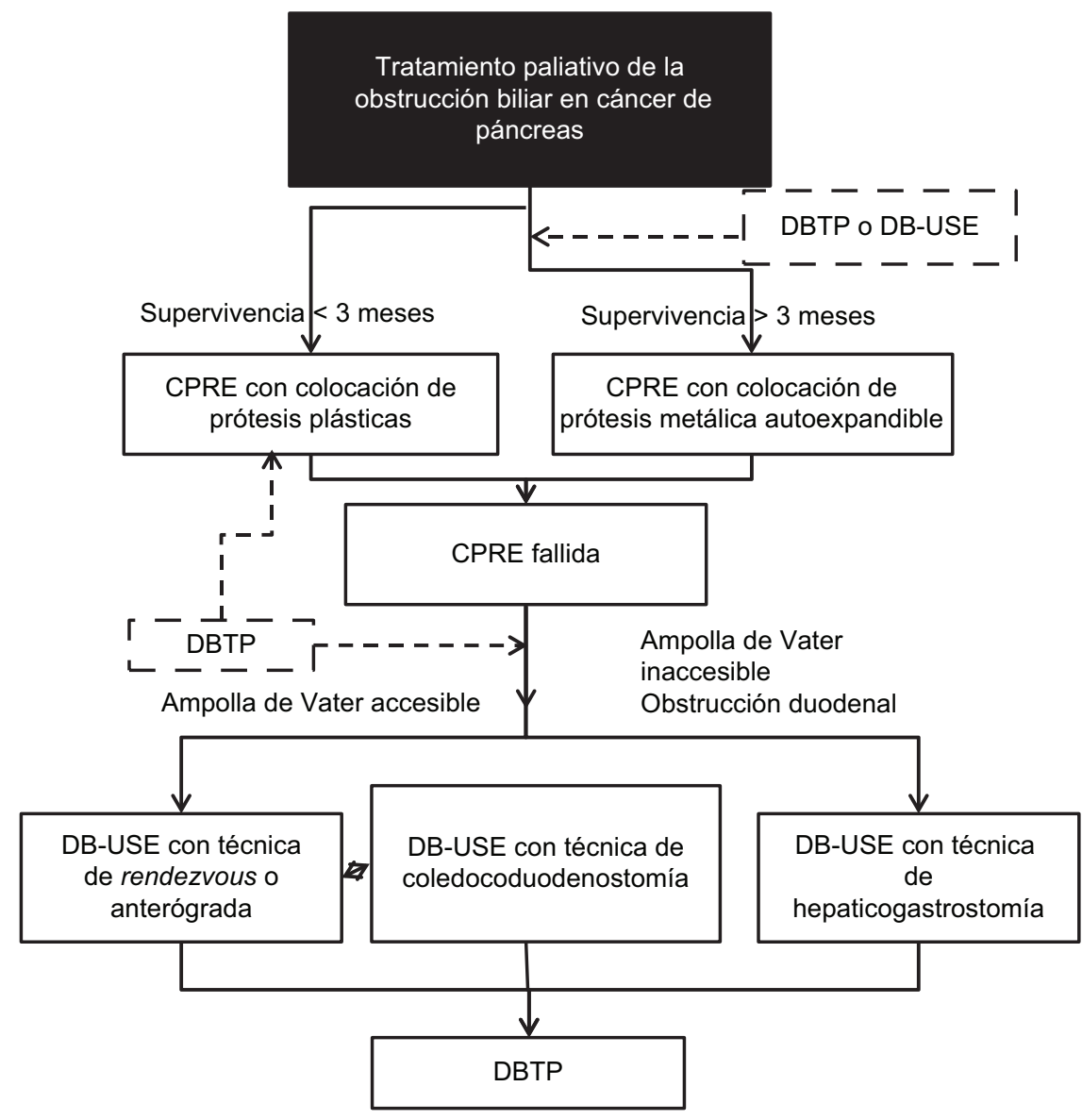

Figura 1. Algoritmo propuesto de tratamiento en obstrucción biliar maligna en pacientes con cáncer de páncreas no resecable.

DBTP: derivación biliar transhepática percutánea; DB-USE: derivación biliar por ultrasonido endoscópico;

CPRE: colangiopancreatografía retrógrada endoscópica.

Además de las técnicas previamente descritas, se ha reportado el tratamiento exitoso de la obstrucción biliar por medio del drenaje transmural de la vesícula biliar, aunque en pacientes con cáncer de páncreas, ha sido solo en series de $\operatorname{casos}^{49}$.

Finalmente, se ha propuesto el uso de DB-USE como tratamiento inicial de la obstrucción biliar distal maligna. Mientras que el éxito clínico y técnico al compararse con la CPRE son similares ${ }^{16,17,19,50-53}$, el DBUSE parece estar asociado a menores eventos adver$\operatorname{sos}^{50}$, particularmente el desarrollo de pancreatitis posprocedimiento ${ }^{19,51-53}$, menor disfunción de la prótesis y necesidad de reintervención ${ }^{51}$. En el estudio de Tellez-Ávila FI., et al. ${ }^{18}$, la DB-USE parece ser más costo efectiva comparada con la CPRE en pacientes con obstrucción biliar distal maligna ${ }^{18}$. Además, con el advenimiento de las prótesis de aposición luminal, la seguridad del procedimiento parece haberse incrementado ${ }^{48}$. El principal factor limitante es que la DB-USE es un procedimiento complejo, por lo que debe ser practicado en centros de endoscopia con experiencia ${ }^{33,54}$.

La CPRE con colocación de prótesis metálicas autoexpandibles representa el estándar de tratamiento de la obstrucción maligna en cáncer de páncreas no resecable, ya que comparado con la cirugía y el drenaje percutáneo presenta menor morbilidad. Así mismo, la DB-USE debe preferirse como tratamiento de rescate y es probable que en un futuro sea considerado como tratamiento de primera línea (Fig. 1).

\section{Bibliografía}

1. Ryan DP, Hong TS, Bardeesy N. Pancreatic adenocarcinoma. N Engl J Med. 2014;371(11):1039-49.

2. Kleeff J, Korc M, Apte M, La Vecchia C, Johnson CD, Biankin AV, et al. Pancreatic cancer. Nat Rev Dis Primers. 2016;2:16022. 
3. Moffat GT, Epstein AS, O'Reilly EM. Pancreatic cancer-A disease in need Optimizing and integrating supportive care. Cancer. 2019;125(22):3927-35

4. Moss AC, Morris E, Mac Mathuna P. Palliative biliary stents for obstructing pancreatic carcinoma. Cochrane Database Syst Rev. 2006(2):CD004200.

5. Arguedas MR, Heudebert GH, Stinnett AA, Wilcox CM. Biliary stents in malignant obstructive jaundice due to pancreatic carcinoma: a cost-effectiveness analysis. Am J Gastroenterol. 2002;97(4):898-904.

6. Glazer ES, Hornbrook MC, Krouse RS. A meta-analysis of randomized trials: immediate stent placement vs. surgical bypass in the palliative management of malignant biliary obstruction. J Pain Symptom Manage. 2014:47(2):307-14.

7. Duan F, Cui L, Bai Y, Li X, Yan J, Liu X. Comparison of efficacy and complications of endoscopic and percutaneous biliary drainage in malignant obstructive jaundice: a systematic review and meta-analysis. Cancer Imaging. 2017;17(1):27

8. Zhao XQ, Dong JH, Jiang K, Huang XQ, Zhang WZ. Comparison of percutaneous transhepatic biliary drainage and endoscopic biliary drainage in the management of malignant biliary tract obstruction: a meta-analysis. Dig Endosc. 2015;27(1):137-45.

9. Abraham NS, Barkun JS, Barkun AN. Palliation of malignant biliary obstruction: a prospective trial examining impact on quality of life. Gastrointest Endosc. 2002;56(6):835-41.

10. Saleem A, Leggett CL, Murad MH, Baron TH. Meta-analysis of randomized trials comparing the patency of covered and uncovered self-expandable metal stents for palliation of distal malignant bile duct obstruction. Gastrointest Endosc. 2011;74(2):321-7.

11. Almadi MA, Barkun A, Martel M. Plastic vs. self-expandable metal stents for palliation in malignant biliary obstruction: A series of meta-analyses. Am J Gastroenterol. 2017;112(2):260-73.

12. Martinez JM, Anene A, Bentley TG, Cangelosi MJ, Meckley LM, Ortendahl JD, et al. Cost effectiveness of metal stents in relieving obstructive jaundice in patients with pancreatic cancer. J Gastrointest Cancer. 2017;48(1):58-65.

13. Deprez PH, Moreels TG, Aouattah T, Piessevaux H, Perez-Cuadrado-Robles $\mathrm{E}$. A new 12-French plastic stent for unresectable distal malignant biliary obstruction. Endoscopy. 2020;52(6):474-82.

14. Pinol V, Castells A, Bordas JM, Real MI, Llach J, Montana X, et al. Percutaneous self-expanding metal stents versus endoscopic polyethylene endoprostheses for treating malignant biliary obstruction: randomized clinical trial. Radiology. 2002;225(1):27-34.

15. Tellez-Avila FI, Herrera-Mora D, Duarte-Medrano G, Lopez-Arce G, Lindoro-Barraza D, Casanova I, et al. Biliary Drainage in Patients With Failed ERCP: Percutaneous Versus EUS-guided Drainage. Surg Laparosc Endosc Percutan Tech. 2018;28(3):183-7.

16. Paik WH, Lee TH, Park DH, Choi JH, Kim SO, Jang S, et al. EUS-guided biliary drainage versus ERCP for the primary palliation of malignant biliary obstruction: A multicenter randomized clinical trial. Am J Gastroenterol. 2018;113(7):987-97.

17. Bang JY, Navaneethan U, Hasan M, Hawes $R$, Varadarajulu S. Stent placement by EUS or ERCP for primary biliary decompression in pancreatic cancer: a randomized trial (with videos). Gastrointest Endosc. 2018;88(1):9-17.

18. Tellez-Avila FI, Figueredo-Zacarias MA, Munoz-Anaya E, Rodriguez-Sanchez JF, Ramirez-Garcia J, Ramirez-Luna M, et al. EUS-guided biliary drainage in patients with distal malignant biliary obstruction requires fewer interventions and has a lower cost compared to ERCP biliary drainage. Surg Endosc. 2020

19. Hathorn KE, Bazarbashi AN, Sack JS, McCarty TR, Wang TJ, Chan WW et al. EUS-guided biliary drainage is equivalent to ERCP for primary treatment of malignant distal biliary obstruction: a systematic review and meta-analysis. Endosc Int Open. 2019;7(11):E1432-E41.

20. Davids PH, Groen AK, Rauws EA, Tytgat GN, Huibregtse K. Randomised trial of self-expanding metal stents versus polyethylene stents for distal malignant biliary obstruction. Lancet. 1992;340(8834-8835):1488-92.

21. Bang BW, Jeong S, Lee DH, Lee JI, Lee SC, Kang SG. The biodurability of covering materials for metallic stents in a bile flow phantom. Dig Dis Sci. 2012:57(4):1056-63.

22. Tringali A, Hassan C, Rota M, Rossi M, Mutignani M, Aabakken L. Covered vs. uncovered self-expandable metal stents for malignant distal biliary strictures: a systematic review and meta-analysis. Endoscopy. 2018;50(6): $631-41$

23. Kitano M, Yamashita Y, Tanaka K, Konishi H, Yazumi S, Nakai Y, et al. Covered self-expandable metal stents with an anti-migration system improve patency duration without increased complications compared with uncovered stents for distal biliary obstruction caused by pancreatic carcinoma: a randomized multicenter trial. Am J Gastroenterol. 2013;108(11): 1713-22.

24. Isayama H, Komatsu $\mathrm{Y}$, Tsujino $\mathrm{T}$, Sasahira N, Hirano K, Toda N, et al. A prospective randomised study of "covered" versus "uncovered" diamond stents for the management of distal malignant biliary obstruction. Gut. 2004;53(5):729-34

25. Isayama H, Kawakubo K, Nakai Y, Inoue K, Gon C, Matsubara S, et al. A novel, fully covered laser-cut nitinol stent with antimigration properties or nonresectable distal malignant biliary obstruction: a multicenter feasibility study. Gut Liver. 2013;7(6):725-30

26. Hamada $T$, Isayama $H$, Nakai $Y$, Iwashita $T$, Ito $Y$, Mukai $T$, et al. Antireflux covered metal stent for nonresectable distal malignant biliary obstruction: Multicenter randomized controlled trial. Dig Endosc. 2019;31(5): 566-74.

27. Mukai $\mathrm{T}$, Yasuda I, Isayama $\mathrm{H}$, Iwashita $\mathrm{T}$, Itoi $\mathrm{T}$, Kawakami $\mathrm{H}$, et al. Pilot study of a novel, large-bore, fully covered self-expandable metallic stent for unresectable distal biliary malignancies. Dig Endosc. 2016;28(6):671-9.

28. Jang SI, Lee SJ, Jeong S, Lee $\mathrm{DH}, \mathrm{Kim} \mathrm{MH}$, Yoon $\mathrm{HJ}$, et al. Efficacy of a multiplex paclitaxel emission stent using a Pluronic $((\mathrm{R}))$ mixture membrane versus a covered metal stent in malignant biliary obstruction: A prospective randomized comparative study. Gut Liver. 2017;11(4):567-73.

29. Inoue T, Suzuki Y, Okumura F, Naitoh I, Sano H, Ibusuki M, et al. Intraductal placement of a fully covered metal stent with a long string for distal malignant biliary obstruction without endoscopic sphincterotomy: Prospective multi-center feasibility study. Dig Endosc. 2020 Jan 3. doi: 10.1111/den.13614. Online ahead of print.

30. Sofi AA, Khan MA, Das A, Sachdev M, Khuder S, Nawras A, et al. Radiofrequency ablation combined with biliary stent placement versus stent placement alone for malignant biliary strictures: a systematic review and meta-analysis. Gastrointest Endosc. 2018;87(4):944-51.

31. Kallis Y, Phillips N, Steel A, Kaltsidis H, Vlavianos P, Habib N, et al. Analysis of endoscopic radiofrequency ablation of biliary malignant strictures in pancreatic cancer suggests potential survival benefit. Dig Dis Sci. 2015;60(11):3449-55.

32. Enochsson L, Swahn F, Arnelo U, Nilsson M, Lohr M, Persson G. Nationwide, population-based data from 11,074 ERCP procedures from the Swedish Registry for Gallstone Surgery and ERCP. Gastrointest Endosc. 2010;72(6):1175-84

33. Holt BA, Hawes R, Hasan M, Canipe A, Tharian B, Navaneethan U, et al. Biliary drainage: role of EUS guidance. Gastrointest Endosc. 2016;83(1):160-5.

34. Artifon EL, Aparicio D, Paione JB, Lo SK, Bordini A, Rabello C, et al. Biliary drainage in patients with unresectable, malignant obstruction where ERCP fails: endoscopic ultrasonography-guided choledochoduodenostomy versus percutaneous drainage. J Clin Gastroenterol. 2012;46(9):768-74.

35. Moole $\mathrm{H}$, Bechtold ML, Forcione D, Puli SR. A meta-analysis and systematic review: Success of endoscopic ultrasound guided biliary stenting in patients with inoperable malignant biliary strictures and a failed ERCP. Medicine (Baltimore). 2017:96(3):e5154.

36. Baniya R, Upadhaya S, Madala S, Subedi SC, Shaik Mohammed T, Bachuwa G. Endoscopic ultrasound-guided biliary drainage versus percutaneous transhepatic biliary drainage after failed endoscopic retrograde cholangiopancreatography: a meta-analysis. Clin Exp Gastroenterol. 2017;10:67-74.

37. Miller CS, Barkun AN, Martel M, Chen YI. Endoscopic ultrasound-guided biliary drainage for distal malignant obstruction: a systematic review and meta-analysis of randomized trials. Endosc Int Open. 2019;7(11): E1563-E73.

38. Khashab MA, Valeshabad AK, Afghani E, Singh VK, Kumbhari V, Messallam $A$, et al. A comparative evaluation of EUS-guided biliary drainage and percutaneous drainage in patients with distal malignant biliary obstruction and failed ERCP. Dig Dis Sci. 2015;60(2):557-65.

39. Hindryckx $P$, Degroote $H$, Tate DJ, Deprez $\mathrm{PH}$. Endoscopic ultrasound-guided drainage of the biliary system: Techniques, indications and future perspectives. World J Gastrointest Endosc. 2019;11(2):103-14.

40. Artifon EL, Marson FP, Gaidhane M, Kahaleh M, Otoch JP. Hepaticogastrostomy or choledochoduodenostomy for distal malignant biliary obstruction after failed ERCP: is there any difference? Gastrointest Endosc. 2015;81(4):950-9.

41. Tellez-Avila FI, Duarte-Medrano G, Gallardo-Cabrera V, Casasola-Sanchez L, Valdovinos-Andraca F. Endoscopic ultrasonography-guided transhepatic antegrade self-expandable metal stent placement in a patient with surgically altered anatomy. Endoscopy. 2015;47(Suppl 1):E643-4.

42. Dhindsa BS, Mashiana HS, Dhaliwal A, Mohan BP, Jayaraj M, Sayles H, et al. EUS-guided biliary drainage: A systematic review and meta-analysis. Endosc Ultrasound. 2020;9(2):101-9.

43. Khashab MA, van der Merwe S, Kunda R, El Zein MH, Teoh AY, Marson FP, et al. Prospective international multicenter study on endoscopic ultrasound-guided biliary drainage for patients with malignant distal biliary obstruction after failed endoscopic retrograde cholangiopancreatography. Endosc Int Open. 2016;4(4):E487-96.

44. Uemura RS, Khan MA, Otoch JP, Kahaleh M, Montero EF, Artifon ELA. EUS-guided choledochoduodenostomy versus hepaticogastrostomy: A systematic review and meta-analysis. J Clin Gastroenterol. 2018;52(2):123-30.

45. Khan MA, Akbar A, Baron TH, Khan S, Kocak M, Alastal Y, et al. Endoscopic ultrasound-guided biliary drainage: A systematic review and meta-analysis. Dig Dis Sci. 2016;61(3):684-703.

46. Kawakubo K, Isayama $H$, Kato H, Itoi T, Kawakami H, Hanada K, et al. Multicenter retrospective study of endoscopic ultrasound-guided biliary 
drainage for malignant biliary obstruction in Japan. J Hepatobiliary Pancreat Sci. 2014;21(5):328-34.

47. Ramirez-Luna MA, Tellez-Avila FI, Giovannini M, Valdovinos-Andraca F, Guerrero-Hernandez I, Herrera-Esquivel J. Endoscopic ultrasound-guided biliodigestive drainage is a good alternative in patients with unresectable cancer. Endoscopy. 2011;43(9):826-30.

48. Krishnamoorthi R, Dasari CS, Thoguluva Chandrasekar V, Priyan $\mathrm{H}$ Jayaraj M, Law J, et al. Effectiveness and safety of EUS-guided choledochoduodenostomy using lumen-apposing metal stents (LAMS): a systematic review and meta-analysis. Surg Endosc. 2020;34(7):2866-77.

49. Chang JI, Dong E, Kwok KK. Endoscopic ultrasound-guided transmura gallbladder drainage in malignant obstruction using a novel lumen-apposing stent: a case series (with video). Endosc Int Open. 2019;7(5):E655-E61.

50. Park JK, Woo YS, Noh DH, Yang Jl, Bae SY, Yun HS, et al. Efficacy of EUS-guided and ERCP-guided biliary drainage for malignant biliary obstruction: prospective randomized controlled study. Gastrointest Endosc. 2018;88(2):277-82.
51. Jin Z, Wei $Y$, Lin $H$, Yang J, Jin H, Shen $S$, et al. Endoscopic ultrasound-guided versus endoscopic retrograde cholangiopancreatography-guided biliary drainage for primary treatment of distal malignant biliary obstruction: A systematic review and meta-analysis. Dig Endosc. 2020;32(1):16-26

52. Han SY, Kim SO, So H, Shin E, Kim DU, Park DH. EUS-guided biliary drainage versus ERCP for first-line palliation of malignant distal biliary obstruction: A systematic review and meta-analysis. Sci Rep. 2019;9(1):16551.

53. Bishay K, Boyne D, Yaghoobi M, Khashab MA, Shorr R, Ichkhanian $Y$ et al. Endoscopic ultrasound-guided transmural approach versus ERCP-guided transpapillary approach for primary decompression of malignant biliary obstruction: a meta-analysis. Endoscopy. 2019;51(10): 950-60.

54. Cotton PB, Eisen G, Romagnuolo J, Vargo J, Baron T, Tarnasky P, et al. Grading the complexity of endoscopic procedures: results of an ASGE working party. Gastrointest Endosc. 2011;73(5):868-74. 